\title{
Population based cost utility study of interferon beta-1b in secondary progressive multiple sclerosis
}

\author{
Raeburn B Forbes, Ann Lees, Norman Waugh, Robert J Swingler
}

\begin{abstract}
Objective To evaluate the cost utility of interferon beta-1b in secondary progressive multiple sclerosis. Design Population based cost utility model (healthcare perspective). Data on use of health services were obtained from case records and routine morbidity data and utility values from a EuroQol survey. Local and published costs were used. Effectiveness was modelled using data on relative risk reductions from a randomised trial of interferon beta- $1 b$.

Setting Tayside region, 1993-5.

Subjects 132 ambulatory people with secondary progressive multiple sclerosis.

Main outcome measures Cost per quality adjusted life year (QALY) gained. Rate of relapse and proportion becoming wheelchair dependent over three years.

Results The number needed to treat for 30 months to delay time to wheelchair dependence in one person by nine months was 18 (95\% confidence interval 5 to 26). For every 18 people treated for 30 months, six relapses would be prevented, gaining 0.397 discounted QALYs. The cost per QALY gained was $£ 1024667$ (£276 466 to £1 485499 ). If treatment was restricted to patients attending neurology services, the number needed to treat was 14 (cost per QALY gained $£ 833514$ (£161358 to $\infty)$ ). The cost per QALY gained was not sensitive to changes in cost which took account of a societal perspective.

Conclusions The cost per QALY gained from interferon beta is high because of the high drug cost and modest clinical effect. Resources could be used more efficiently elsewhere.
\end{abstract}

\section{Introduction}

Interferon beta is the first treatment to alter the natural course of relapsing-remitting multiple sclerosis. ${ }^{1-3}$ In a recent trial involving 718 people the drug was also shown to modify the secondary progressive form of the disease, reducing relapse rates over a 30 month follow up. ${ }^{4}$ Furthermore, the proportion of interferon beta- $1 b$ recipients who became wheelchair dependent was reduced by $32 \%$, and this benefit lasted for nine months. ${ }^{4}$ A lack of effect on progression of disability was mooted as a reason for not making interferon beta- $1 b$ (which costs over $£ 9600$ a year for each patient) more widely available to patients with relapsing-remitting multiple sclerosis in the United Kingdom. ${ }^{5}$ The disease course is different in secondary progressive multiple sclerosis, ${ }^{6}$ so evidence from trials in relapsing-remitting multiple sclerosis cannot be extrapolated to patients with secondary progressive disease.

The results of the recent trial in secondary progressive multiple sclerosis ${ }^{4}$ will increase demand for interferon beta-1b, placing further pressure on limited

\section{What is cost utility analysis?}

The main benefit of a healthcare intervention should be greater "health." A potential measure of "health" is the quality adjusted life year (QALY). To calculate a QALY, the duration of health state (in years) is multiplied by a factor representing the quality ("utility") of that health state. The quality (or utility) value for economic evaluation is usually derived from a health index. In health indices, health is rated along an interval scale, where 1 equals perfect health and 0 represents dead. It is also possible to rate health states with a negative value--that is worse than death. Values for health states are usually derived using time-trade off methods or standard gamble techniques. Studies which calculate the cost per QALY gained from an intervention are called cost utility analyses.

Values for the cost per QALY gained across a range of different interventions can be used to inform resource allocation decisions. There are arguments for limiting access to interventions which have a very high cost per QALY, as there may be alternative uses for those funds which are more efficient-for example, they provide benefits to more people. Conversely, fewer restrictions might be placed on interventions that have a low cost per QALY gained.

budgets. As resources are limited, spending on treatments such as interferon beta means foregoing benefits from other forms of care-this is known as the opportunity cost. ${ }^{7}$ To allow comparison of the relative merits of alternative options we conducted a cost utility analysis of treatment with interferon beta- $1 \mathrm{~b}$ in secondary progressive multiple sclerosis based on the benefits shown in the recent trial (box). ${ }^{4}$

\section{Methods}

We compared the effect of treating a cohort of people with secondary progressive multiple sclerosis with interferon beta for 30 months against existing best practice without interferon beta. Ideally, we would have used effectiveness data derived from a meta-analysis of clinical trials, but only one completed trial exists with data in the public domain. Therefore, our model represents a best case scenario based on current knowledge (box). We estimated the costs and benefits of a programme of treatment in sufficient people to postpone wheelchair dependence in one person. Although we took a health service perspective, we allowed for a societal perspective through use of sensitivity analysis.

\section{Study population}

We aimed to identify all patients with multiple sclerosis in Tayside in order to have a representative cohort. Residents of Tayside region (population 395 600) with multiple sclerosis were identified from four sources (neurology department records, visual evoked response requests, Scottish morbidity records, and a
Department of

Neurology,

Ninewells Hospital

and Medical School,

Dundee DD1 9SY

Raeburn B Forbes

research fellow

Robert J Swingler

consultant neurologist

Argyll and Clyde

Health Board,

Paisley PA2 7BN

Ann Lees

health economist

Scottish Health

Purchasing

Information Centre,

Aberdeen

AB15 6RE

Norman Waugh

director

Correspondence to:

R B Forbes,

Department of

Neurology, Royal

Victoria Hospital,

Belfast BT12 6BA

raeburn.forbes@

royalhospitals.

n-i-nhs.uk

BMJ 1999;319:1529-33 
Formula for calculating cost per QALY gained by treatment with interferon beta- $1 \mathrm{~b}$ in secondary progressive multiple sclerosis

Cost per QALY gained $=$ net cost of treatment/number of QALYs gained where

Net cost of treatment $=$ total cost of treatment - costs averted (from reduced relapses and wheelchair dependence)

Total cost of treatment $=$ number needed to treat $(\mathrm{NNT}) \times 30$ months $\times$ unit cost of interferon beta- $1 \mathrm{~b}$ $\mathrm{NNT}=$ Number needed to treat to delay time to wheelchair dependence by nine months

survey of the region's general practitioners). Diagnosis was confirmed from hospital or primary care case records. ${ }^{8}$ Capture-recapture methods indicate that our point estimate of prevalence on 1 September 1996 was $94 \%$ complete. ${ }^{9}$ Using accepted definitions, ${ }^{6}$ we identified people with secondary progressive disease on the basis of the most recent information in their case records.

Patients who had diagnosed secondary progressive disease on 1 January 1993 formed the cohort for this study (population cohort). To explore the effect of limiting prescription to ambulatory patients with more active disease, a subset of the population cohort who were either admitted to the neurology unit or referred to neurology outpatient clinics at Tayside hospitals during January 1993 to June 1993 were identified (neurology subset). In Tayside, people with secondary progressive multiple sclerosis are not routinely followed up but are referred to the outpatient clinic for specialist management of new problems such as relapses or other chronic symptoms. All of the cohort was followed up until 31 December 1995.

\section{Relapse rates and wheelchair dependence}

We used the same definitions of relapse as the clinical trial. ${ }^{4}$ Admissions for treatment of a relapse and the year in which patients became wheelchair dependent were identified from hospital case records. Ideally, we would have calculated the proportion who became wheelchair dependent within 30 months (the same follow up as the trial ${ }^{4}$ ), but the case records lacked detail. Thus the number needed to treat to delay time to wheelchair dependence could be an underestimate.

We identified episodes of oral corticosteroid prescription from the University of Dundee's medicines monitoring unit prescribing database using a record linkage method. ${ }^{10}$ We assumed that each corticosteroid prescription represented a community treated relapse. Prescriptions associated with bron-

Table 1 Nine month costs (1995 prices) to health service and society of patients with multiple sclerosis ${ }^{14}$ and estimate of cost of extra nine months of wheelchair dependence

\begin{tabular}{|c|c|c|}
\hline & $\begin{array}{l}\text { Cost to health } \\
\text { service }(£)\end{array}$ & Cost to society* $(£)$ \\
\hline Able to walk unaided for unlimited distances & 262 & 2322 \\
\hline Able to walk limited distances unaided or with walking stick & 494 & 6570 \\
\hline Need to use wheelchair on most/every day & 3334 & 11723 \\
\hline Cost of additional 9 months of wheelchair dependence & 2840 & 5153 \\
\hline
\end{tabular}

*Excludes transfer costs. Transfer costs include benefit payments and are excluded from economic analyses, although often included in cost of illness studies. chodilators were assumed to be for asthma and were excluded. We calculated the rate of relapse requiring hospital admission and the rate of community treated relapse and estimated confidence intervals using a method for proportions. ${ }^{11}$ A previous study of relapse frequency in secondary progressive multiple sclerosis showed a reduction in relapse from the first year after onset to the second year. ${ }^{12}$ Although we could have used these data in our model, our population cohort contained people who had had the disease for more than two years and were likely to have a lower relapse rate. A recent survey of British neurologists indicated that $47 \%$ of neurologists treated at least half of multiple sclerosis relapses with corticosteroids. ${ }^{13}$ Therefore, we assumed that for every relapse treated (hospital or community) there was another relapse which was not treated.

\section{Costs saved by delaying time to wheelchair dependence and prevention of relapses}

We estimated the costs saved by delaying time to wheelchair dependence by nine months using data from a cost analysis based on information from 672 members of the Multiple Sclerosis Society of Great Britain and Northern Ireland (table 1). ${ }^{14}$ Other estimates of the cost of multiple sclerosis to the United Kingdom did not have sufficient detail for our model. ${ }^{15}{ }^{16}$ We identified resources used during hospital treated relapses in Tayside and valued them using cost data from local NHS trust finance directorates (Robert Hudson, personal communication). We distinguished between fixed and non-fixed costs when costing relapses, but this was not possible for wheelchair dependence as the study ${ }^{14}$ lacked that information. We assumed that each community treated relapse required 20 minutes' consultation with a general practitioner ${ }^{17}$ and that untreated relapses did not incur health service costs (although general practitioner costs may have been incurred). The cost of corticosteroids was taken from the 1995 British National Formulary. ${ }^{18}$ All costs were adjusted to a 1995 price base using the hospital and community health services price index (Department of Health, London).

\section{Calculation of quality adjusted life years}

To determine current health we conducted a postal survey of people with multiple sclerosis in Tayside in Spring 1998 (three years after the end of follow up) after obtaining permission from general practitioners. This survey created a baseline from which to estimate the effect of interferon beta. Respondents indicated their current level of ambulation on a postal ambulation scale (based on the scale of McAlpine and Compston $^{19}$ ) and completed the EuroQol (EQ-5D) health related quality of life instrument. ${ }^{20}$ Responses from people with secondary progressive multiple sclerosis were selected. We obtained values for EQ-5D health states from published social tariffs using mean valuations based on 10 year trade off values. ${ }^{21}$ The EQ-5D was chosen as it is simple to complete and has been extensively tested in UK populations. In an unpublished survey of 67 Tayside residents we had previously established that that increasing postal ambulation scale scores accurately identified people with increasing severity of multiple sclerosis and that appropriate changes in utility values were occurring. 
The number of QALYs gained by postponing wheelchair dependence by nine months was equal to the change in EQ-5D value associated with a change in postal ambulation scale score from 4 to 5 (table 2) multiplied by 0.75 years. The number of QALYs gained by preventing a one month hospital relapse was equal to the change in EQ-5D value associated with a change in postal ambulation scale score from 4 to 5 multiplied by 0.083 years. For each community treated and untreated relapse, postal ambulation scale scores changed from 3 to 4 for one month. We assumed that $40 \%$ of QALYs were gained in years 1 and 2 and 20\% were gained in the final six months of the 30 month programme. A discount rate of $6 \%$ was applied to QALYs gained in years 2 and 3 .

\section{Cost utility ratios}

Assuming a $32 \%$ relative reduction in the proportion of people who become wheelchair dependent during follow up, we calculated the number needed to treat ${ }^{22}$ for 30 months to postpone wheelchair dependence by nine months for one person; $95 \%$ confidence intervals for the numbers needed to treat were calculated from the $95 \%$ confidence limits for the absolute risk reduction and expressed as number needed to treat (harm) to number needed to treat (benefit). ${ }^{23}$ In our cost utility model, interferon beta- $1 \mathrm{~b}$ cost $£ 800$ per patient per month. ${ }^{18}$ Other costs associated with interferon beta treatment were not included as we had no reliable estimate from our local practice. Using trial data we estimated that $12 \%$ of hospital relapses would be prevented, that corticosteroid use would decrease by $21 \%$, and that $31 \%$ of untreated relapses would be prevented. ${ }^{4}$ Treatment costs in the second and third years were discounted at a rate of $6 \%$ (UK treasury rate). Upper and lower limits of the cost utility ratios were estimated using the $95 \%$ confidence intervals of the number needed to treat.

\section{Sensitivity and threshold analyses}

A sensitivity analysis explored the effect of $(a)$ increasing the cost of each additional nine months of wheelchair dependence (taking account of a societal perspective), (b) changes in the unit cost of interferon beta-1b, $(c)$ changes in the number of, and discount rate applied to, QALYs gained, or (d) assuming that the relative reduction in hospital admission and corticosteroid use was $31 \%$ (not $12 \%$ or $21 \%$ ). Assuming that an acceptable threshold of cost utility would be $£ 10000$ per QALY gained, ${ }^{24}$ we determined the changes required to model variables to breach this threshold. We also performed threshold analyses at $£ 20000, £ 30000$, and $£ 50000$ per QALY gained.

\section{Results}

We identified 756 people with multiple sclerosis in Tayside during 1993-5. Of these, 178 had secondary progressive disease, 46 of whom were already wheelchair dependent. There were 132 people in the population cohort, and 17 people in the neurology subset.

\section{Wheelchair dependence}

Twenty four $(18 \%)$ of the population cohort became wheelchair dependent within 36 months of follow up.
Table 2 Mean utility value (health state value) for people with secondary progressive multiple sclerosis stratified by postal ambulation scale score

\begin{tabular}{|c|c|c|c|}
\hline Postal ambulation scale score (descriptor) & $\begin{array}{c}\text { No of } \\
\text { patients }\end{array}$ & Mean (SD) utility & Change in utility \\
\hline 1 (Unrestricted) & 2 & 1.00 & \\
\hline 2 (Up to $500 \mathrm{~m}$ unaided) & 1 & 0.620 & 0.380 \\
\hline 3 (Up to $250 \mathrm{~m}$ with aid) & 9 & $0.643(0.087)$ & -0.023 \\
\hline 4 (Walk indoors with assistance) & 20 & $0.389(0.343)$ & 0.254 \\
\hline 5 (Wheelchair dependent) & 46 & $0.015(0.31)$ & 0.374 \\
\hline 6 (Restricted to bed) & 6 & $-0.260(0.24)$ & 0.275 \\
\hline
\end{tabular}

Delaying progression from postal ambulation scale 4 to 5 by 9 months gained $0.281(0.374 \times 0.75)$ QALYs; $0.031(0.374 \times 0.083)$ QALYs are gained by preventing a one month hospital treated relapse and 0.021 $(0.254 \times 0.083)$ QALYs for each untreated or community treated relapse prevented.

We estimated that the number needed to treat with interferon beta-1b for 30 months to postpone wheelchair dependence by nine months was 18 (95\% confidence interval 5 to 26 ). Four (24\%) of the neurology subset became wheelchair dependent. The number needed to treat to postpone wheelchair dependence by nine months in the neurology subset was 14 (number needed to treat (harm) 9 to $\infty$ to number needed to treat (benefit) 3).

\section{Relapse rates}

We identified 32 admissions for treatment of a relapse in the 36 month follow up period, giving a rate of 0.08 (95\% confidence interval 0.06 to 0.11 ) per patient-year. A total of 56 discrete corticosteroid episodes were identified in 21 people. Three people had concomitant bronchodilators prescribed, so their steroids were assumed to be for asthma. One oral steroid prescription preceded an admission to hospital for relapse by 15 days; all other episodes of community and hospital treated relapses were mutually exclusive. The rate of community treated relapses was $0.15(0.11$ to 0.18 ) per patient-year. In the 30 months to the end of December 1995, the neurology subset experienced seven hospital admissions for relapse and four community corticosteroid prescriptions and therefore an estimated 11 untreated relapses.

\section{Quality adjusted life years gained}

We sent a postal survey to 569 people with multiple sclerosis, of whom $402(71 \%)$ returned valid responses. Of these 402, 84 had secondary progressive disease. Using these 84 responses and a discount rate of $6 \%$ for benefits, treatment of 18 members of the population cohort and 14 members of the neurology subset for 30 months would result in a gain of 0.397 and 0.357 QALYs respectively.

\section{Cost utility ratios}

Table 3 shows the main values used to calculate the cost utility ratios. The net cost per QALY gained from interferon beta-1b in secondary progressive multiple sclerosis was $£ 1024393$ (95\% confidence interval $£ 276191$ to $£ 1484824$ ). For the neurology subset, the cost per QALY gained was $£ 883209$ (£179 209 to $\infty$ ).

\section{Sensitivity and threshold analyses}

A 190\% increase in the cost of each additional nine months of wheelchair dependence from $£ 2840$ to $£ 5153$ (which takes account of a societal perspective) reduced the cost per QALY gained by only $0.2 \%$. A $25 \%$ increase in the number of QALYs gained in 
Table 3 Inputs to the cost utility model of interferon beta $1 \mathrm{~b}$ for secondary progressive multiple sclerosis (Tayside 1993-5)

\begin{tabular}{|c|c|c|}
\hline & $\begin{array}{l}\text { Population } \\
\text { cohort }\end{array}$ & $\begin{array}{c}\text { Neurology } \\
\text { subset }\end{array}$ \\
\hline $\begin{array}{l}\text { No needed to treat for } 30 \text { months to delay time to wheelchair } \\
\text { dependence by } 9 \text { months for } 1 \text { person }\end{array}$ & 18 & 14 \\
\hline No of hospital treated relapses prevented ${ }^{\star}$ & 1 & 1 \\
\hline No of community treated relapses prevented $†$ & 2 & 1 \\
\hline No of untreated relapses prevented & 3 & 2 \\
\hline Cost saved per hospital treated relapse prevented & $£ 1700$ & $£ 2130$ \\
\hline Costs saved per community treated relapse prevented & $£ 30$ & $£ 30$ \\
\hline QALYs gained per 9 months of wheelchair dependence avoided & 0.281 & 0.281 \\
\hline QALYs gained per hospital treated relapse prevented & 0.031 & 0.031 \\
\hline QALYs gained per community treated or untreated relapse prevented & 0.021 & 0.021 \\
\hline Relative risk reduction of wheelchair dependence ${ }^{4}$ & $32 \%$ & $32 \%$ \\
\hline Relative risk reduction of hospital admission ${ }^{4}$ & $12 \%$ & $12 \%$ \\
\hline Relative risk reduction in corticosteroid use ${ }^{4}$ & $21 \%$ & $21 \%$ \\
\hline Relative risk reduction in untreated relapses ${ }^{4}$ & $31 \%$ & $31 \%$ \\
\hline
\end{tabular}

${ }^{*}$ As there was a $12.1 \%$ relative reduction in hospital admissions in the trial, ${ }^{4}$ one $(0.49$ rounded up to nearest integer) hospital admission would have been prevented for every 18 people treated for 30 months. †As there was a $21 \%$ relative reduction in corticosteroid use in the trial, ${ }^{4} 2(1.38$ rounded up to nearest integer) community treated relapses would have been prevented for every 18 people treated for 30 months.

secondary progressive multiple sclerosis (to 0.496 discounted QALYs) reduced the cost per QALY gained in the population cohort by $20 \%$ (to $£ 819514$ ). If the unit cost of interferon beta- $1 \mathrm{~b}$ was reduced by half to $£ 4800$ per patient a year, the cost per QALY gained fell by $49.4 \%$ to $£ 506407$. If we assumed that $31 \%$ of hospital admissions and community treated relapses were prevented (in the trial $31 \%$ of all relapses were prevented, but only $12 \%$ of hospital admissions and $21 \%$ of steroid use was prevented ${ }^{4}$ ) the cost per QALY gained was $£ 832399$ ( $£ 221831$ to $£ 1208133$ ) in the population cohort and $£ 628797$ ( $£ 122079$ to $\infty$ ) in the neurology subset. None of the proposed thresholds could be breached without extreme changes to the model's variables (table 4).

\section{Discussion}

The recent trial showing benefit from interferon beta in secondary progressive multiple sclerosis ${ }^{4}$ has led to a call for the treatment to be made available immediately to all patients with that form of the disease. ${ }^{25}$ The Multiple Sclerosis Society of Great Britain and Northern Ireland issued minimum standards of care for people with multiple sclerosis ${ }^{26}$ and is planning a national campaign to raise the profile of the disease in the United Kingdom. ${ }^{27}$ Despite the need for improvements in the care of people with multiple sclerosis, our analysis shows that treatment with interferon beta- $1 \mathrm{~b}$ has a significant opportunity cost and resources could probable be used better elsewhere.

\section{Robustness of cost utility model}

Studies that take a health service perspective are often criticised for ignoring social costs of disease. This is particularly relevant to multiple sclerosis, as healthcare expenditure is typically $15 \%$ to $20 \%$ of the total cost of the disease..$^{15}{ }^{15}$ However, our cost utility ratio was unaffected by changes in the cost of care, and arguments that interferon beta- $1 \mathrm{~b}$ might have an acceptable cost utility ratio if social costs were considered $^{29}$ now seem implausible. Other countries have estimated similar costs for nine additional months of severe disability (Belgium: 9200 ecu (£6345) health and social service perspective ${ }^{30}$; United States $\$ 2911$ (£1712) healthcare perspective ${ }^{31}$; and Canada \$11 427 (£4395) societal perspective ${ }^{28}$. This model should be valid outside the United Kingdom unless the cost of an additional nine months of wheelchair dependence and six relapses exceeds $£ 400000$.

The high cost utility ratio was driven by the cost of interferon beta- $1 \mathrm{~b}$ and the modest clinical effect. The cost of interferon beta- $1 \mathrm{~b}$ will probably fall over time because of competition from other products, ${ }^{32}$ but price reductions are unlikely to be sufficient to make its use cost effective (table 4).

\section{Comparisons with other healthcare interventions}

Treatment of secondary progressive multiple sclerosis with interferon beta-1b is one of the least efficient interventions for improving health in a population. We identified 104 citations on Medline using the terms "cost" and "quality adjusted life year" from 1995 to October 1998. The median cost per QALY from the 128 cost utility ratios cited was $£ 15625$ (interquartile range $£ 9167$ to $£ 74969$ ) and only one other study exceeded $£ 1$ million per QALY gained. ${ }^{33}$ Although comparisons of the cost per QALY gained may conceal important methodological differences ${ }^{34}$ Richards was probably correct to conclude that most competing demands for healthcare funds are likely to produce greater health gains than interferon beta for multiple sclerosis. ${ }^{5}$

\section{Alternative uses for funds for interferon beta-1b}

Our cost utility ratios are of a similar size to estimates in studies of relapsing-remitting multiple sclerosis$£ 328000$ to $£ 809000$ per QALY in Britain ${ }^{35}$ and $\$ 300000$ in Canada. ${ }^{36}$ Although targeting treatment at patients with more active disease seemed more efficient than treating all patients with secondary progressive disease, the opportunity cost was still substantial.

The health related quality of life of people with multiple sclerosis is much lower than that of the

Table 4 Threshold analysis of interferon beta-1b treatment for secondary progressive multiple sclerosis (Tayside, 1993-5): values required to meet different costs per QALY thresholds

\begin{tabular}{|c|c|c|c|c|}
\hline & $\begin{array}{l}\text { Cost per QALY gained } \\
\text { (£) }\end{array}$ & $\begin{array}{l}\text { Yearly cost of interferon/patient }(£) \\
(\% \text { reduction from baseline })\end{array}$ & $\begin{array}{l}\text { people treated for } 30 \text { months } \\
\text { (increase from baseline) }\end{array}$ & $\begin{array}{l}\text { wheelchair dependence avoided }(£) \\
\text { (increase from baseline) }\end{array}$ \\
\hline Baseline & 1024393 & 9600 (baseline) & 0.397 (baseline) & 2840 (baseline) \\
\hline Threshold 1 & 50000 & $565(94)$ & 8.2 (21-fold) & 389975 (150-fold) \\
\hline Threshold 2 & 30000 & $379(96)$ & 13.6 (34-fold) & 397900 (153-fold) \\
\hline Threshold 3 & 20000 & 287 (97) & 20.3 (51-fold) & 402000 (155-fold) \\
\hline Threshold 4 & 10000 & $194(98)$ & 40.8 (103-fold) & 405850 (156-fold) \\
\hline
\end{tabular}


Key messages

- Secondary progressive multiple sclerosis is a potentially disabling disorder associated with low health related quality of life

- Interferon beta-1b may reduce rate of relapse

- The benefits of interferon beta- $1 \mathrm{~b}$ treatment are very low relative to its cost

- Cost utility analysis estimated a cost of over $£ 1 m$ per QALY gained

- Cost per QALY was not affected by taking into account the costs of care

- Money would be better spent on other ways of improving quality of life than on interferon beta

general adult population, ${ }^{37}$ matched controls with other chronic diseases, ${ }^{38}$ and controls with another neurological disorder (epilepsy). ${ }^{39}$ Therefore, interventions which improve the quality of life of people with multiple sclerosis more efficiently than interferon beta-1b need to be identified. It is probably appropriate to allocate more resources to people with secondary progressive multiple sclerosis, but access to interferon beta-1b should be restricted. Realistically, far more benefit would be obtained from directing funds into improved supportive care.

We thank the medicines monitoring unit (MEMO) of the University of Dundee; R Hudson, D Carson, M Foster, and L Dick of the Scottish Health Purchasing Information Centre costing unit; Shirley Macdonald; and Andrew Owen.

Contributors: RBF and RJS had the idea for the study. RBF, AL, NW and RJS contributed to study design. RBF was responsible for data collection, performed the analysis of results, and wrote the first draft of the manuscript. AL, NW, and RJS al edited and approved the final version of the manuscript. RBF and RJS are guarantors of the study.

Funding: None.

Competing interests: RBF and RJS were investigating and evaluating neurologists for a clinical trial of Antegren in multiple sclerosis, sponsored by Athena Neurosciences. RBF has received funding to attend a conference from Serono. RJS has received grants from Serono, Schering Healthcare, and Biogen to fund attendance at conferences.

1 IFNB Multiple Sclerosis Study Group. Interferon beta1b is effective in relapsing-remitting multiple sclerosis. I. Clinical results of a multicenter, randomised, double blind, placebo controlled trial. Neurolog, $1993 ; 43: 655-61$

2 Jacobs LD, Cookfair DL, Rudick RR, Herndon RM, Richet JR, Salazir AM, et al. Intramuscular interferon beta 1a for disease progression in relapsing multiple sclerosis. Ann Neurology 1996;39:285-94

3 PRISMS Study Group. Randomised double-blind placebo controlled study of interferon beta 1a in relapsing/remitting multiple sclerosis. Lancet 1998;352:1498-1504.

4 European Study Group on Interferon beta-1b in Secondary Progressive Multiple Sclerosis. Placebo-controlled multicentre randomised trial of interferon beta-1b in treatment of secondary progressive multiple sclerosis. Lancet 1998;352:1491-7.

5 Richards RG. Interferon beta in multiple sclerosis. Clinical cost effectiveness falls at the first hurdle. BMJ 1996:313:1159.

6 Lublin FD, Reingold SC. Defining the clinical course of multiple sclerosis: results of an international survey. Neurology 1996;46:907-11.

7 Mooney G. Economics, medicine and health care. 2nd ed. Hemel Hempstead: Prentice Hall Europe, 1992:5-20.

8 Poser CM, Paty DW, Scheinberg L, McDonald I, Davis FA, Ebers GC, et al. New diagnostic criteria for multiple sclerosis: guidelines for research protocols. Ann Neurol 1983;13:227-31.

9 Forbes RB, Swingler RJ. Estimating the prevalence of multiple sclerosis in the United Kingdom using capture recapture methodology. Am J Epidemiol 1999;149:1016-24.

10 Evans JMM, McDevitt DG, MacDonald TM. The Tayside medicines monitoring unit (MEMO): a record-linkage system for pharmacovigilance. Pharmaceutical Med 1995;9:177-84.

11 Gardner MJ, Altman DG. Calculating confidence intervals for proportions and their differences. In: Statistics with confidence. London: BMJ, 1989:28-33.
12 Weinshenker BG, Bass B, Rice GPA, Noseworthy J, Carriere W, Baskerville J, et al. The natural history of multiple sclerosis: a geographically based study. 2. Predictive value of the early clinical course. Brain $1989 ; 112: 1419-28$.

13 Tremlett HL, Luscombe DK, Wiles CM. Use of corticosteroids in multiple sclerosis by consultant neurologists in the United Kingdom. J Neurol Neurosurg Psychiatry 1998;65:362-5.

14 Holmes J, Madgwick T, Bates D. The cost of multiple sclerosis. Br J Med Econ 1995;8:181-93.

15 Blumhardt L, Wood C. The economics of multiple sclerosis: a cost of illness study. Br J Med Econ 1996;10:99-118.

16 O'Brien B. Multiple sclerosis. London: Office of Health Economics, 1987.

17 Graham B, McGregor K. What does a GP consultation cost? Br J Gen Prac 1997;47:170-2.

18 British Medical Association, Royal Pharmaceutical Society of Great Britain. British national formulary. Vol 30. London: BMA, RPSGB, 1995.

19 McAlpine D, Compston N. Some aspects of the natural history of disseminated sclerosis. QJ Med 1952;21:135-67.

20 EUROQOL Group. EuroQol-a new facility for the measurement of health related quality of life. Health Policy 1990;16:199-208.

21 Dolan P, Gudex C, Kind P, Williams A. A social tariff for EuroOol:results from a UK general population survey. York: Centre for Health Economics, University of York, 1995. (Discussion paper 138.)

22 Sackett DL, Richardson WS, Rosenberg WMC, Haynes RB. How to practice and teach evidence based medicine. London: Churchill Livingstone, 1996.

23 Altman DG. Confidence intervals for number needed to treat. BMJ 1998;317:1309-12

24 Laupacis A, Feeny D, Detsky AS, Tugwell PX. How attractive does a new technology have to be to warrant adoption and utilization? Tentative guidelines for using clinical and economic evaluations. Can Med Ass J 1992;146:473-81.

25 Goodkin DE. Interferon beta therapy for multiple sclerosis. Lance 1998;352:1486-7.

26 Freeman J, Johnson J, Rollinson S, Thompson A, Hatch J. Standards of healthcare for people with MS. London: Multiple Sclerosis Society of Great Britain and Northern Ireland, Neurorehabilitation and Therapy Services Directorate of the National Hospital for Neurology and Neurosurgery, Director

27 Speak out: moving MS into the millennium. MS Matters 1998 Nov/Dec:12-3.

28 Canadian Burden of Illness Study Group. Burden of illness of multiple sclerosis. 1. Cost of illness. Can J Neurol Sci 1998;25:23-30.

29 Holmes J. Avoidance of need for social care should also be taken into account. BMJ 1997:314:601.

30 Carton H, Loos R, Pacolet J, Versieck K, Vlietinck R. Utilisation and cost of professional care and assistance according to disability of patients with multiple sclerosis in Flanders (Belgium). J Neurol Neurosurg Psychiatr 1998;64:444-50.

31 Stolp-Smith KA, Atkinson EJ, Campion ME, O'Brien PC, Rodriguez M. Health care utilization in multiple sclerosis. A population based study in Olmstead County, MN. Neurology 1998;50:1594-1600.

32 Tolley KH, Whynes DK. Interferon-b in multiple sclerosis. Can we control its costs? Pharmacoeconomics 1997;11:210-5.

33 Busch MP, Dodd RY, Lackritz EM, AuBuchon JP, Birkmeyer JD, Petersen JD. Value and cost-effectiveness of screening blood donors for antibody to hepatitis B core antigen as a way of detecting window-phase human mmunodeficiency virus type 1 infections. The HIV Blood Donor Study Group. Transfusion 1997;37:1003-11.

34 Gerard K, Mooney G. OALY league tables: handle with care. Health Econ 1993;2:59-64.

35 Parkin D, McNamee P, Jacoby A, Miller P, Thomas S, Bates D. A cost utility study of interferon beta for multiple sclerosis. Health Technol Assessment 1998:2(4)

36 Brown MG, Murray TJ, Fisk JD, Sketris IS, Schwartz CE, Blanc JCL. $A$ therapeutic and economic assessment of Betaferon in multiple sclerosis. Halifax: Dalhousie University 1996.

37 Vickrey BG, Hays RD, Harooni R, Myers LW, Ellison GW. A health related quality of life measure for multiple sclerosis. Quality Life Res 1995;4:187206.

38 Holmes J, Young C, Reti M. Quality of life in multiple sclerosis. J Neurol Neurosurg Psychiatry 1998;64:420.

39 Vickrey BG, Hays RD, Genovese BJ, Myers LW, Ellison GW. Comparison of generic to disease-targeted health related quality of life measures for multiple sclerosis. J Clin Epidemiol 1997;50:5457-69.

(Accepted 24 August 1999)

\section{Endpiece \\ Wise advice}

Those who think they have not time for bodily exercise will sooner or later have to find time for illness.

Edward Stanley, 1826-93, at an address at Liverpool College, 20 December 1873

Submitted by T O Cheng, professor of medicine, Washington DC 